\title{
Tipificación molecular de Mycobacterium avium subsp. paratuberculosis en lecherías de Antioquia, Colombia
}

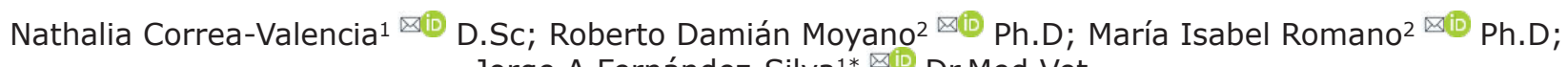
Jorge A Fernández-Silva ${ }^{*} \bowtie \mathbb{B}$ Dr.Med.Vet.

1 Universidad de Antioquia, Facultad de Ciencias Agrarias, Escuela de Medicina Veterinaria, Grupo Centauro, Medellín, Colombia. 2Institute of Agrobiotechnology and Molecular Biology (IABIMO) INTA-CONICET. De Los Reseros y Las Cabañas S/N, Hurlingham, Buenos Aires, Argentina.

${ }^{*}$ Correspondencia: jorge.fernandez@udea.edu.co

Recibido: Octubre 2019; Aceptado: Mayo 2020; Publicado: Agosto 2020.

\section{RESUMEN}

Objetivo. Determinar la diversidad molecular de Mycobacterium avium subsp. paratuberculosis (MAP) en muestras ambientales de hatos lecheros colombianos. Materiales y métodos. Las muestras ambientales de 25 hatos lecheros positivos a MAP por IS900-qPCR se cultivaron por duplicado en medio de yema de huevo de Herrold con micobactina J para obtener aislamientos. Las colonias sospechosas fueron confirmadas para MAP por IS900-qPCR. EI ADN positivo se subtipó utilizando técnicas de unidades micobacterialess repetitivas intercaladas - número variable de repeticiones en tándem (MIRU-VNTR) y técnicas de repeticiones de multilocus de secuencia corta (MLSSR) para analizar las diferencias genéticas entre los aislamientos. Resultados. El subtipado reveló dos genotipos diferentes por MIRU-VNTR (INMV 2 e INMV 36). La técnica de MLSSR se realizó para aumentar el poder discriminatorio de lo obtenido por MIRU-VNTR, pero no se observaron diferencias entre los aislamientos recuperados. Conclusiones. El presente estudio representa un enfoque importante para el conocimiento del estatus epidemiológico de MAP en la población de estudio.

Palabras clave: Genética; genotipado; enfermedad de Johne; MLSSR; MIRU-VNTR (Fuente: National Agricultural Library Thesaurus).

\section{ABSTRACT}

Objective. To determine Mycobacterium avium subsp. paratuberculosis (MAP) molecular diversity in environmental samples from Colombian dairy herds. Materials and methods. Environmental samples from 25 IS900-qPCR MAP-positive dairy herds were cultured by duplicate in Herrold's egg yolk medium with mycobactin J to obtain isolates. Suspicious colonies were confirmed by MAPIS900-qPCR. Positive DNA was sub-typed using mycobacterial interspersed repetitive units-variable number of tandem repeat (MIRU-VNTR) and multilocus short sequence repeats (MLSSR) techniques to analyze the genetic differences between the isolates. Results. Sub-typing revealed two different genotypes by MIRU-VNTR (INMV 2 and INMV 36). MLSSR technique was carried out to increase the

Como citar (Vancouver).

Correa-Valencia NM, Moyano RD, Romano MI, Fernández-Silva JA. Tipificación molecular de Mycobacterium avium subsp. paratuberculosis en lecherías de Antioquia Colombia. Rev MVZ Córdoba. 2020; 25(3):e1816. https://doi.org/10.21897/rmvz.1816 
discriminatory power from what was obtained by MIRU-VNTR, but no differences were observed among the recovered isolates. Conclusions. The present study represents an important approach to the knowledge on MAP epidemiological status in the study population.

Keywords: Genetics; genotyping; Johne's disease; MLSSR; MIRU-VNTR (Source: National Agricultural Library Thesaurus).

\section{INTRODUCCIÓN}

Mycobacterium avium subsp. paratuberculosis (MAP) es un patógeno que afecta varias especies animales y que, en condiciones in vitro, es dependiente de micobactina, y de crecimiento extremadamente lento. La infección por MAP conduce a una enteritis granulomatosa crónica en animales susceptibles, conocida como paratuberculosis (PTB) o enfermedad de Johne (1). Los signos clínicos de la PTB incluyen diarrea, pérdida de peso, disminución de la producción de leche y carne, lo que lleva a importantes pérdidas económicas (2). Una de las principales preocupaciones acerca de la infección por MAP es la facilidad con que se propaga la bacteria, ya que los animales subclínicos o clínicamente infectados eliminan el agente en las heces y la leche, lo que permite su diseminación a animales jóvenes susceptibles, al medio ambiente y a alimentos para consumo humano (3). La leche y la carne que contienen MAP son especialmente preocupantes ya que se ha sugerido que la bacteria está posiblemente asociada con la enfermedad de Crohn en humanos (4).

La discriminación molecular de MAP a partir de los aislamientos en campo se convierte en una herramienta crucial para complementar el conocimiento general sobre la bacteria, su distribución, comportamiento y características $(5,6)$. El uso de métodos de subtipado molecular de MAP ha aumentado en las últimas dos décadas $(7,8)$. El subtipado de MAP es una herramienta útil en la investigación epidemiológica, que ofrece una mejor comprensión del origen de la infección por MAP, la dinámica, los factores de riesgo asociados, los perfiles de transmisión, la patogénesis, entre otras características relacionadas, lo que permite un diseño racional de medidas de control adecuadas, mejora del diagnóstico y desarrollo de vacunas $(7,9)$. Sin embargo, el subtipado de cepas de MAP representa un desafío ya que son genéticamente monomórficas y las técnicas moleculares tradicionales tienen un poder discriminatorio limitado. Los avances y la disponibilidad de la secuenciación del genoma completo han ampliado las posibilidades para la caracterización de MAP, proporcionando un contexto filogenético que facilita los estudios epidemiológicos globales (10).

Las técnicas de subtipado incluyen técnicas de unidades micobacteriales repetitivas intercaladas-número variable de repeticiones en tándem (MIRU-VNTR), que se basan en el polimorfismo de elementos repetitivos y, técnicas de repeticiones de multilocus de secuencia corta (MLSSR), que consisten en la detección de segmentos más simples de repeticiones en tándem de $2-5 \mathrm{pb}(11,12)$. Se ha reportado que la combinación de métodos dirigidos a diferentes estructuras genómicas de MAP (i.e. MIRU-VNTR, MLSSR) aumenta la capacidad discriminatoria de los métodos combinados en comparación con la capacidad de cada método utilizado por separado $(5,13,14)$.

Con el tiempo se ha logrado cierto conocimiento sobre la PTB en Colombia, sin embargo, la información sobre el impacto y la distribución de la enfermedad, así como de su epidemiología molecular en el país aún es limitada, incluso siendo una enfermedad de reporte obligatorio en el desde $2015(15,16)$. Por lo tanto, el objetivo del presente estudio es determinar la diversidad molecular de MAP en aislamientos a partir de muestras ambientales de hatos lecheros ubicados en la región norte del departamento de Antioquia (Colombia) mediante técnicas de MIRU-VNTR y MLSSR.

\section{MATERIALES Y MÉTODOS}

Hatos de estudio. El presente estudio se realizó en 25 hatos lecheros previamente detectados como infectados por MAP mediante IS900-qPCR en muestras ambientales. Los hatos infectados se detectaron a partir de una población de 386 predios lecheros ubicados en 62 distritos de seis municipios diferentes en la región norte del departamento de Antioquia (Colombia), muestreados entre julio y octubre de 2016. Los 25 hatos de estudio se ubicaron en seis distritos diferentes en tres municipios diferentes. Cada 
hato participante fue visitado una vez durante el período de estudio para recolectar dos muestras ambientales compuestas. El primero contenía material de al menos seis sitios diferentes (submuestras) de concentración de ganado adulto y/o áreas de alto tráfico (e.g. potreros, áreas que rodean bebederos y comederos, callejones, canalones, áreas de espera de salas de ordeño). El segundo contenía material de la sala de ordeño colectada en el tanque estercolero, después de mezclar su contenido durante al menos $5 \mathrm{~min}$. Las seis sub-muestras del segundo lugar se obtuvieron de diferentes lugares del perímetro de la laguna sumergiendo el recipiente de muestreo hasta $10 \mathrm{~cm}$ por debajo de la superficie. Las muestras compuestas definitivas fueron de aproximadamente $20 \mathrm{~g}$, conservadas bajo refrigeración a $4^{\circ} \mathrm{C}$ durante el transporte de regreso al laboratorio $y$, en el laboratorio, se homogeneizaron y luego fueron congeladas a $-20^{\circ} \mathrm{C}$ hasta la extracción de ADN. La extracción de ADN se llevó a cabo utilizando un kit comercial de preparación de ADN (ZR Fecal DNA Kit ${ }^{\top M}$, Zymo Research, CA, EE. UU.). El protocolo incluía un paso previo de disrupción mecánica (Disruptor Genie $^{\circledR} 120$ V, Thomas Scientific, Swedesboro, NJ, EE. UU.). EI ADN extraído se analizó usando un kit comercial de IS900-qPCR dúplex (Bactotype MAP PCR Kit ${ }^{\circledR}$, Qiagen, Leipzig, Alemania).

Cultivo. Las muestras ambientales de los hatos positivos por IS900-qPCR se descontaminaron con solución de cloruro de hexadecilpiridinio (HPC) al $0.75 \%(\mathrm{p} / \mathrm{v})$ durante $24 \mathrm{~h}$, de acuerdo con los procedimientos estándar (15). Los inóculos fueron cultivados en medio de yema de huevo de Herrold (HEYM) con micobactina J por duplicado. Todos los medios de cultivo se incubaron a $37^{\circ} \mathrm{C}$ durante 24 semanas y se verificaron semanalmente para detectar crecimiento de micobacterias o contaminación con microorganismos indeseables. El crecimiento de MAP se monitoreó visualmente para determinar la tasa de crecimiento lenta típica y la morfología de la colonia de acuerdo con descripciones previas (colonias que se desarrollan después de $\geq 3$ semanas de incubación, inicialmente redondas, lisas y blancas, que tienden a acumularse ligeramente y se vuelven de color amarillo claro opaco con arrugas en la superficie). Las colonias se sub-cultivaron en HEYM y aquellas que mostraran un crecimiento compatible con $M A P$, se consideraron como sospechosas. Todos los aislamientos se sometieron a IS900-qPCR confirmatorio (Bactotype MAP PCR Kit ${ }^{\circledR}$, Qiagen, Leipzig, Alemania) para confirmar la identidad de los aislamientos. La descontaminación, así como los procedimientos de cultivo, se llevaron a cabo en la unidad de diagnóstico de la Facultad de Ciencias Agrarias, Universidad de Antioquia, en Medellín (Colombia).

Subtipado de MAP. Para la caracterización molecular de los aislamientos de MAP se aplicó una combinación de dos métodos de subtipado diferentes, ambos basados en la amplificación por PCR de elementos repetitivos del genoma de MAP.

MIRU-VNTR. El procedimiento se llevó a cabo mediante la amplificación de ocho loci MIRUVNTR 3, 7, 10, 25, 32, 47, 292 y X3 (alias 1658), utilizando las condiciones de PCR reportadas previamente (12), con ligeras modificaciones en las condiciones del PCR. La mezcla de PCR estaba compuesta por $2 \mu \mathrm{L}$ de una solución de ADN diluida diez veces, añadida a un volumen final de $50 \mu \mathrm{L}$ que contenía $0.25 \mu \mathrm{L}$ de ADN polimerasa GoTaq $^{\circledR}$ (Promega; $5 \cup / \mu \mathrm{L}$ ), $2 \mu \mathrm{L}$ de betaína (Sigma), dTTP (Qiagen, Leipzig, Alemania), $5 \mu \mathrm{L}$ de tampón de PCR (suministrado por el fabricante), $1 \mu \mathrm{M}$ de cebadores y $2 \mathrm{mM}$ de $\mathrm{MgCl}_{2}$. Las reacciones se llevaron a cabo utilizando un termociclador SimpliAmp (Applied Biosystems, Darmstadt, Alemania). Las condiciones de PCR incluyeron 1 ciclo de $3 \mathrm{~min}$ a $95^{\circ} \mathrm{C}$, 1 ciclo de $1 \mathrm{~min}$ a $95^{\circ} \mathrm{C}, 30$ ciclos de $1 \mathrm{~min} \mathrm{~s}$ a $58^{\circ} \mathrm{C}, 1$ ciclo de $1 \mathrm{~min}$ a $72^{\circ} \mathrm{C}$ y $10 \mathrm{~min}$ a $72^{\circ} \mathrm{C}$. Para determinar el peso molecular de cada producto de PCR y estimar el número de repeticiones en tándem presentes en cada loci, se cargaron 10 $\mu l$ de producto de PCR en un gel de agarosa al $2 \%$, con una escalera de $50 \mathrm{pb}$. Para digitalizar el gel, se utilizó el generador de imágenes Gel Doc TM (BioRad). Los resultados se expresaron mediante un código octal y se determinó el patrón de genotipo (INMV) utilizando la base de datos en línea de acceso libre internacional (http://mac-inmv.tours.inra.fr/). Los genotipos MIRU-VNTR se expresaron como la combinación del número de repeticiones encontradas en cada locus. Se usó la cepa MAP K10 como control de referencia.

MLSSR. El procedimiento se llevó a cabo mediante la amplificación de cuatro loci de repeticiones de secuencia corta (SSR), locus 1 (repeticiones $\mathrm{g}$ ), locus 2 (repeticiones $\mathrm{g}$ ), locus 8 (repeticiones ggt) y locus 9 (repeticiones tgc), utilizando condiciones de PCR como se reportó previamente (11), con ligeras modificaciones en las condiciones del PCR. La selección se realizó seleccionando los loci con el índice discriminatorio más alto (11). La mezcla de PCR 
estaba compuesta de $2 \mu \mathrm{L}$ de una solución de ADN diluida diez veces, añadida a un volumen final de $50 \mu \mathrm{L}$ que contenía $0.5 \mu \mathrm{L}$ de ADN polimerasa GoTaq ${ }^{\circledR}$ (Promega; $5 \mathrm{U} / \mu \mathrm{L}$ ), dTTP (Qiagen, Leipzig, Alemania), $10 \mu \mathrm{L}$ de tampón de PCR (suministrado por el fabricante), $1 \mu \mathrm{M}$ de cebadores y $2 \mathrm{mM}$ de $\mathrm{MgCl}_{2}$. Las reacciones se llevaron a cabo utilizando un termociclador SimpliAmp (Applied Biosystems, Darmstadt, Alemania). Las condiciones de PCR incluyeron 1 ciclo de $3 \mathrm{~min}$ a $95^{\circ} \mathrm{C}, 1$ ciclo de $1 \mathrm{~min}$ a $95^{\circ} \mathrm{C}$, 35 ciclos de 1 min a $60^{\circ} \mathrm{C}, 1$ ciclo de $2 \min$ a $72^{\circ} \mathrm{C}$ y $7 \mathrm{~min}$ a $72^{\circ} \mathrm{C}$. Los productos de PCR se analizaron por electroforesis utilizando geles de agarosa al 1.5\% (grado de electroforesis en agarosa; TransGen Biotech). Todos los amplicones de cada locus se purificaron utilizando el kit de purificación de PCR MinElute ${ }^{\circledR}$ (Qiagen, Leipzig, Alemania) y fueron secuenciados de forma independiente (Unidad de Genómica, Laboratorio de Biotecnología, Consejo Nacional de Investigaciones Científicas y Técnicas, Argentina). La calidad de la secuenciación y el número de unidades de repetición cortas para identificar los alelos se analizaron utilizando el software de análisis de secuencia v5.4 (Applied Biosystems). Los genotipos MLSSR se expresaron como la combinación del número de repeticiones encontradas en los cuatro loci amplificados por PCR. Si el número de repeticiones $\mathrm{g}$ en el locus
2 fue superior a 11 , las repeticiones g para dicho locus se denotaron como $>11 \mathrm{~g}$ como se ha sugerido previamente (13). Los procedimientos de subtipado se llevaron a cabo en el Instituto de Agrobiotecnología y Biología Molecular (IABIMO) INTA-CONICET en Buenos Aires, Argentina.

\section{RESULTADOS}

De las 25 muestras ambientales positivas a MAP por IS900-qPCR, 13 mostraron un crecimiento compatible con MAP en uno o ambos medios HEYM, seis de los cuales fueron confirmados como MAP por IS900-qPCR. Todos los aislamientos crecieron dentro de 8-15 semanas de incubación. En total, tres aislamientos de MAP recuperados de muestras ambientales recolectadas del tanque estercolero de tres hatos diferentes fueron adecuados para el subtipado de MAP. Los tres aislamientos revelaron dos genotipos diferentes por MIRU-VNTR [INMV 2 (código numérico: 32332228) e INMV 36 (código numérico: 32342228)]. MLSSR se llevó a cabo para aumentar el poder discriminatorio, pero no se observaron diferencias, como se puede ver en la Tabla 1. Sin embargo, el perfil MLSSR obtenido del predio 3 puede corresponder a MLSSR 50 (código numérico: 711555455455) de acuerdo con reportes anteriores (13).

Tabla 1. Perfiles de MIRU-VNTR y MLSSR obtenidos a partir de aislamientos ambientales de hatos lecheros positivos a MAP en el departamento de Antioquia, Colombia.

\begin{tabular}{|c|c|c|c|c|c|c|c|c|c|c|c|c|c|c|c|}
\hline \multirow{2}{*}{$\begin{array}{c}\text { Hato/ } \\
\text { Aislamiento }\end{array}$} & \multirow{2}{*}{ Municipio } & \multirow{2}{*}{ Vereda } & \multicolumn{8}{|c|}{ Número de copias de MIRU-VNTR } & \multirow{2}{*}{$\begin{array}{l}\text { Perfil } \\
\text { INMV }\end{array}$} & \multicolumn{4}{|c|}{$\begin{array}{l}\text { Número de copias del } \\
\text { loci SSR }\end{array}$} \\
\hline & & & 3 & 7 & 10 & 25 & 32 & 47 & $292 \times 3$ & $(1658)$ & & $1(g)$ & $2(g)$ & $8(\mathbf{g g t})$ & $9(\operatorname{tgc})$ \\
\hline 1 & Entrerríos & Toruro & 2 & 2 & 2 & 3 & 8 & 4 & 3 & 2 & 36 & 7 & 10 & 5 & 4 \\
\hline 2 & $\begin{array}{l}\text { San Pedro de } \\
\text { los Milagros }\end{array}$ & $\begin{array}{c}\text { Santa } \\
\text { Bárbara }\end{array}$ & 2 & 2 & 2 & 3 & 8 & 4 & 3 & 2 & 36 & 7 & 10 & 5 & 4 \\
\hline 3 & $\begin{array}{l}\text { San Pedro de } \\
\text { los Milagros }\end{array}$ & San Francisco & 2 & 2 & 2 & 3 & 8 & 3 & 3 & 2 & 2 & 7 & 11 & 5 & 4 \\
\hline
\end{tabular}

\section{DISCUSIÓN}

En el presente estudio, se aplicaron las técnicas de subtipado MIRU-VNTR y MLSSR con el objetivo de lograr un análisis epidemiológico de MAP en una de las regiones lácteas más representativas de Colombia. Este país tropical tiene un sistema de producción animal y prácticas de cría que pueden variar en comparación con otras regiones del mundo. Por lo tanto, se espera que la epidemiología molecular de MAP en el país muestre patrones particulares de los de otros países estacionales y tropicales.

Trece de los cultivos mostraron un crecimiento compatible con MAP, siendo seis de ellos confirmados como MAP por IS900-qPCR, y de éstos, tres aislamientos diferentes fueron 
adecuados para el subtipado de MAP. La baja tasa de recuperación de la bacteria pudo deberse al hecho de que las muestras estuvieron en congelación a $-20^{\circ} \mathrm{C}$ durante un máximo de 56 días, lo cual pudo afectar la sensibilidad de la detección (17). De acuerdo con las características reportadas por la literatura en cuanto a color, apariencia y velocidad de crecimiento diferencial de MAP (17), se consideraron aislamientos compatibles, aunque posteriormente no fueron confirmados a partir de la prueba molecular. Lo anterior se puede presentar debido a condiciones desfavorables relacionadas con la calidad del ADN, principalmente como consecuencia de los procesos de transporte y conservación de las muestras, así como de los procesos de extracción de ADN, considerando que las pruebas de tipado utilizadas requieren una calidad definida del ADN de origen (11). Adicionalmente, no se encontró poli-diversidad genética en ninguna de las muestras a partir de las técnicas utilizadas. Esto puede deberse, hipotéticamente, al azar al momento de realizar los lavados de los medios lo cual correspondería a la matriz de análisis.

Aunque las comparaciones con otros estudios son difíciles debido al uso de diferentes loci para el análisis, los perfiles MIRU-VNTR 1 (INMV 1) y 2 (INMV 2) han sido reportados previamente como los genotipos más comunes encontrados en aislamientos de otros países latinoamericanos $(12,18,19,20)$, así como de aislamientos europeos $(5,21,22,23)$. Sin embargo, nuestros hallazgos no se pueden comparar directamente con los reportados previamente (18), ya que consideramos ocho loci MIRU-VNTR y cuatro SSR $y$ en el otro estudio utilizaron 11 loci $[1,3,7,4$, $10,25,32,47,259,292$ y X3 -alias 1658] y tres SSR [1, 2 y 8 , respectivamente.

Por otro lado, algunos reportes que utilizan los mismos loci/SSR se han publicado previamente. El perfil de INMV 2 fue reportado en un aislamiento a partir de materia fecal de ganado bovino (24). Se reportaron siete perfiles diferentes de INMV, incluido el INMV 2, en bovinos y caprinos de aislamientos argentinos y mexicanos (19). El INMV 2 se reportó como el segundo perfil más común encontrado en heces y/o mucosa intestinal de bovinos y humanos en Argentina, también concluyendo que INMV 2 fue el clon original del que derivan los otros (20). De acuerdo con los perfiles MLSSR reportados en el presente estudio, los subtipos aislados en nuestro estudio se encuentran comúnmente en bovinos y otras especies en diferentes países $(13,18,25)$. Curiosamente, un aislamiento bovino del país vecino de Colombia, Venezuela, ha mostrado un genotipo diferente por SSR (11g-10g-5ggt5ggt), lo que sugiere diversidad de cepas en la parte norte de América del Sur (13).

A una escala nacional, se reportó una caracterización molecular por MIRU-VNTR y MLSSR de ocho aislamientos de MAP (obtenidos de materia fecal, tejidos y tanques estercoleros) de cinco hatos lecheros en la región norte de Antioquia, ya definidos como infectados por MAP según pruebas directas e indirectas (18). Los autores revelaron dos perfiles de cepas combinadas diferentes ( $1 \mathrm{~A}$ y $2 \mathrm{~B}$ ), siendo la primera caracterización molecular de MAP en Colombia hasta esa fecha. Los hallazgos presentados aquí no pueden compararse con los reportados previamente en el país (18), ya que consideramos ocho loci MIRU-VNTR y utilizaron doce $[1,2,3,4,7,10,25,32,47,259,292$ y X3 -alias 1658]. Sin embargo, los autores consideraron los mismos cuatro SSR, reportando el genotipo MLSSR B [7-g, 10-g, 5-ggt, 4-tgc], obtenido a partir de muestras de tejidos y de tanques estercoleros.

Como conclusión, y en base a los patrones de subtipado de los aislamientos de MAP obtenidos, a partir de 386 hatos lecheros ubicados en la región lechera del norte de Antioquia (Colombia), encontramos diferentes genotipos de MAP que circulan en la región de estudio: INMV 2 e INMV 36 según el análisis MIRU-VNTR. Los aislamientos con INMVs idénticos, provenientes de dos municipios distintos, no pudieron ser discriminados mediante MLSSR. Los hallazgos de este estudio conducen a importantes implicaciones epidemiológicas con respecto al control y prevención de PTB en Colombia. Se sugiere que los métodos de tipificación más nuevos, como el polimorfismo de un solo nucleótido (SNP), que es capaz de detectar diferencias entre los tipos principales, se pueden utilizar para obtener un mayor discernimiento de las características epidemiológicas de MAP en el país, incluida la influencia de los medios de cultivo, el papel desempeñado por la vida silvestre local, la diversidad de los agroecosistemas y el cruce de animales importados e indígenas a ser tomados en cuenta en el análisis como posibles fuentes de diversidad genómica de MAP. 


\section{Conflicto de interés}

Los autores no tienen conocimiento de ninguna relación financiera o personal con otras personas u organizaciones que puedan influir de manera inapropiada en el trabajo que se informa en este documento. Los patrocinadores del estudio (CODI- Universidad de Antioquia) no tuvieron un papel directo en el desarrollo del diseño del estudio, el análisis o la interpretación de la recopilación de datos.

\section{Agradecimientos}

Los autores agradecen a todo el equipo de investigación que realizó todos los procedimientos de prueba y diagnóstico, y a los productores cuyas propiedades fueron seleccionadas en este estudio, por su buena voluntad y paciencia. Este trabajo fue apoyado por la Convocatoria Programática 2014-2015: Área de Ciencias de la Salud, Universidad de Antioquia (Beca \# 87142015-2042).

\section{REFERENCIAS}

1. Harris NB, Barletta RG. Mycobacterium avium subsp. paratuberculosis in veterinary medicine. Clin Microbiol Reviews. 2001; 14:489-512. https://doi.org/10.1128/ CMR.14.3.489-512.2001

2. McAloon CG, Whyte P, More SJ, Green MJ, O'Grady L, Garcia A, et al. The effect of paratuberculosis on milk yield-A systematic review and meta-analysis. J Dairy Sci. 2016; 99(2):1449-1460. https://doi.org/10.3168/ jds.2015-10156

3. Sweeney RW, Collins MT, Koets AP, Mcguirk SM, Roussel AJ. Paratuberculosis (Johne's disease) in cattle and other susceptible species. J Vet Intern Med. 2012; 26(6):12391250. https://doi.org/10.1111/j.19391676.2012.01019.x

4. Kuenstner JT, Naser S, Chamberlin W, Borody T, Graham DY, McNees A, et al. The Consensus from the Mycobacterium avium ssp. paratuberculosis (MAP) Conference 2017. Front Public Heal. 2017; 5:1-5. https://doi.org/10.3389/fpubh.2017.00208

5. Douarre PE, Cashman W, Buckley J, Coffey A, O'Mahony J. Molecular characterization of Mycobacterium avium subsp. paratuberculosis using multi-locus short sequence repeat (MLSSR) and mycobacterial interspersed repetitive unitsvariable number tandem repeat (MIRUVNTR) typing methods. Vet Microbiol. 2011; 149(3-4):482-487. https://doi. org/10.1016/j.vetmic.2010.12.001
6. Rónai Z, Csivincsik A, Gyuranecz M, Kreizinger $Z$, Dán A, Jánosi $S$. Molecular analysis and MIRU-VNTR typing of Mycobacterium avium subsp. paratuberculosis strains from various sources. J Appl Microbiol. 2015; 118(2):275-283. https://doi.org/10.1016/j. meegid.2016.03.004.

7. Motiwala AS, Li L, Kapur V, Sreevatsan S. Current understanding of the genetic diversity of Mycobacterium avium subsp. paratuberculosis. Microbes Infect. 2006; 8(5):1406-1418. https://doi.org/10.1016/j. micinf.2005.12.003

8. Sohal JS, Singh S V., Subodh S, Sheoran $\mathrm{N}$, Narayanasamy K, Singh PK, et al. Mycobacterium avium subspecies paratuberculosis diagnosis and geno-typing: Genomic insights. Microbiol Res. 2009; 164(3):330-337. https://doi.org/10.1016/j. micres.2007.03.005

9. Sohal JS, Singh $S V$, Singh $P K$, Singh $A$ V. On the evolution of "Indian Bison type" strains of Mycobacterium avium subspecies paratuberculosis. Microbiol Res. 2010; 165(2):163-171. https://doi. org/10.1016/j.micres.2009.03.007

10. Ahlstrom C, Barkema HW, De Buck J, Ahlstrom C, Barkema HW, Buck J De, et al. Relative frequency of 4 major strain types of Mycobacterium avium ssp. paratuberculosis in Canadian dairy herds using a novel single nucleotide polymorphism-based polymerase chain reaction. J Dairy Sci. 2016; 99(10):8297-8303. https://doi. org/10.3168/jds.2016-11397 
11. Amonsin $A$, Li LL, Zhang $Q$, Bannantine JP, Motiwala AS, Sreevatsan S, et al. Multilocus short sequence repeat sequencing approach for differentiating among Mycobacterium avium subsp. paratuberculosis strains 3. J Clin Microbiol. 2004; 42(0095-1137):1694-1702. https:// doi.org/10.1128/JCM.42.4.1694-1702.2004

12. Thibault VC, Grayon M, Boschiroli $M L$, Hubbans C, Overduin P, Stevenson K, et al. New variable-number tandem-repeat markers for typing Mycobacterium avium subsp. paratuberculosis and M. avium strains: Comparison with IS900 and IS1245 restriction fragment length polymorphism typing. J Clin Microbiol. 2007; 45(8):2404-2410. https:// doi.org/10.1128/JCM.00476-07

13. Thibault VC, Grayon M, Boschiroli $M L$, Willery $\mathrm{E}$, Allix-Béguec $C$, Stevenson $\mathrm{K}$, et al. Combined multilocus short-sequencerepeat and mycobacterial interspersed repetitive unit-variable-number tandemrepeat typing of Mycobacterium avium subsp. paratuberculosis isolates. J Clin Microbiol. 2008; 46(12):4091-4094. https://doi.org/10.1128/JCM.01349-08

14. Castellanos E, Aranaz A, Gould KA, Linedale R, Stevenson K, Alvarez J, et al. Discovery of stable and variable differences in the Mycobacterium avium subsp. paratuberculosis type I, II, and III genomes by pan-genome microarray analysis. Appl Environ Microbiol. 2009; 75(3):676-686. https://doi.org/10.1128/AEM.01683-08

15. Correa-Valencia, NM, García-Tamayo Y, Fernández-Silva JA. Mycobacterium avium subsp. paratuberculosis in Colombia (1924-2016): A review. Rev Colomb Cienc Pecu. 2018; 31(3):165-179. https://doi. org/10.17533/udea.rccp.v31n3a01

16. Whittington $R$, Donat $K$, Weber MF, Kelton D, Nielsen SS, Eisenberg S, et al. Control of paratuberculosis: who, why and how. A review of 48 countries. BMC Vet Res. 2019; 15:198. https://doi.org/10.1186/s12917-019-1943-4

17. Whittington R. Cultivation of Mycobacterium avium Subsp. Paratuberculosis. in: Paratuberculosis: Organism, Disease, Control. CAB International, Oxfordshire, England; 2010.

18. Fernández-Silva JA, Abdulmawjood $A$, Bülte M. Diagnosis and molecular characterization of Mycobacterium avium subsp. paratuberculosis from dairy cows in Colombia. Vet Med Int. 2011; 2011:1-12. https://doi.org/10.4061/2011/352561
19. Fernández-Silva JA, Abdulmawjood A, Akineden Ö, Bülte M. Genotypes of Mycobacterium avium subsp. paratuberculosis from South American countries determined by two methods based on genomic repetitive sequences. Trop Anim Health Prod. 2012; 44(6):1123-1126. https://doi.org/10.1007/ s11250-011-0060-6

20. Gioffré $A$, Muñoz MC, Alvarado Pinedo MF, Vaca R, Morsella C, Fiorentino MA, et al. Molecular typing of Argentinian Mycobacterium avium subsp. paratuberculosis isolates by multiple-locus variable number-tandem repeat analysis. Brazilian J Microbiol. 2015; 46(2):557-564. https://doi.org/10.1590/ $\underline{\mathrm{S} 1517-838246220140283}$

21. Imperiale $B R$, Moyano RD, Di Giulio $A B$, Romero MA, Alvarado Pinedo MF, Santangelo $\mathrm{MP}$, et al. Genetic diversity of Mycobacterium avium complex strains isolated in Argentina by MIRU-VNTR. Epidemiol Infect. 2017; 145(7):1382-1391. https://doi. org/10.1017/S0950268817000139

22. Stevenson K, Alvarez J, Bakker D, Biet F, De Juan L, Denham S, et al. Occurrence of Mycobacterium avium subspecies paratuberculosis across host species and European countries with evidence for transmission between wildlife and domestic ruminants. BMC Microbiol. 2009; 9:1-13. https://doi.org/10.1186/1471-2180-9-212

23. Biet F, Sevilla IA, Cochard T, Lefrançois LH, Garrido JM, Heron I, et al. Interand Intra-subtype genotypic differences that differentiate Mycobacterium avium subspecies paratuberculosis strains. BMC Microbiol. 2012; 12(1):264. https://doi. org/10.1186/1471-2180-12-264

24. de Kruijf $M$, Lesniak ON, Yearsley $D$, Ramovic E, Coffey A, O'Mahony J. Low genetic diversity of bovine Mycobacterium avium subspecies paratuberculosis isolates detected by MIRU-VNTR genotyping. Vet Microbiol. 2017; 203:280-285. https://doi. org/10.1016/j.vetmic.2017.03.029

25. Correa M, Medina G, Rentería T. Caracterización molecular de Mycobacterium avium subespecie paratuberculosis en bovinos y ovinos de Mexicali, Baja California, México. Rev Mex Cienc Pecu. 2013; 4(4):489500. https://cienciaspecuarias.inifap.gob. $\underline{\mathrm{mx} / \text { index.php/Pecuarias/article/view/3202 }}$ 